\title{
Avaliação dos modelos logístico bicompartimental e de Gompertz na estimativa da dinâmica de fermentação ruminal in vitro do farelo e da torta de babaçu (Orbignya martiana)
}

[Avaliation of bicompartimental logistic and Gompertz mathematical models to estimate gas production from babassu (Orbignya martiana) meal and pie using the semi-automated in vitro technique]

\author{
L.N. Farias ${ }^{1}$, V.R. Vasconcelos ${ }^{1}$, F.F.R. Carvalho ${ }^{2}$, J.L.R. Sarmento ${ }^{3}$ \\ ${ }^{1}$ Universidade Federal do Piauí \\ 64049-550 - Teresina, PI \\ ${ }^{2}$ Universidade Federal Rural de Pernambuco - Recife, PE \\ ${ }^{3}$ Universidade Federal do Piauí - Bom Jesus, PI
}

\begin{abstract}
RESUMO
Utilizaram-se dois modelos matemáticos para avaliar a produção de gases do farelo e da torta de babaçu, pela técnica in vitro semiautomática de produção de gases. Foram utilizados o modelo logístico e o de Gompertz. Os parâmetros de validação usados foram o quadrado médio do erro (QME), o coeficiente de determinação $\left(\mathrm{R}^{2}\right)$, o desvio médio absoluto dos resíduos (DMA) e a análise gráfica dos resíduos. $\mathrm{O}$ modelo logístico bicompartimental apresentou menores valores $(\mathrm{P}<0,05)$ para o QME e o DMA em relação ao de Gompertz, e não houve diferença $(\mathrm{P}>0,05)$ quanto ao $\mathrm{R}^{2}$. Os gráficos de dispersão mostraram semelhanças nos ajustes dos dois modelos. Na análise gráfica dos resíduos, os dois modelos descreveram bem cinética de produção de gases da matéria seca. No entanto, o modelo logístico apresentou melhor valor de QME. Para avaliação da cinética de fermentação ruminal do farelo e da torta de babaçu pela técnica in vitro semiautomática de produção de gases, recomenda-se adotar o modelo logístico.
\end{abstract}

Palavras-chave: produção de gases, inóculo ruminal, modelos matemáticos

\begin{abstract}
Two mathematical models were used to evaluate gas production from the meal and pie of babassu using the semi-automated gas production technique. The logistic and Gompertz models were used and the validation parameters for both models were the residual mean square (RMS), the coefficient of determination $\left(R^{2}\right)$, the absolute average residual (AAR), and the graphical analysis of residues. The logistic model showed lower RMS $(P<0.05)$ and AAR in comparison to Gompertz model. Both Gompertz and logistic models showed similar $R^{2}$. The dispersion graphics showed similarity for both models and graphics analyses demonstrated that both models describe well the kinetic of gas production of dry matter. However, the bicompartimental logistic model showed the best RMS value. Logistic models are recommended to describe the kinetic of gas production from babassu foods using the semi-automated in vitro technique.
\end{abstract}

Keywords: gas production, ruminal inoculums, mathematical models

\section{INTRODUÇÃo}

A eficiência de produção de ruminantes é, em grande parte, atribuída ao balanceamento adequado da ração. Formulações eficientes e econômicas dependem do valor nutritivo dos

Recebido em 20 de julho de 2009

Aceito em 28 de dezembro de 2010

E-mail: luciananfvet@yahoo.com.br ingredientes. Portanto, novas metodologias de avaliação de alimentos que possam ser rápidas, pouco onerosas e precisas têm sido constantemente buscadas.

A técnica in vitro semiautomática de produção de gases tem sido utilizada para predizer a 
digestibilidade in vivo. Tal técnica tem por objetivo simular as condições normais do rúmen, como atmosfera anaeróbica, temperatura de incubação constante $\left(39^{\circ} \mathrm{C}\right)$ e $\mathrm{pH}$ ótimo (Maurício et al., 2003; Noguera et al., 2004). Este método pode ser utilizado para determinar as taxas de degradação dos alimentos por monitoramento das taxas de produção de gases durante determinados intervalos de tempo (Beuvink e Kogut, 1993; Jayme et al., 2009). Como principais vantagens, a técnica apresenta baixo custo, possibilita a avaliação dos diferentes ambientes do rúmen e da taxa de fermentação dos constituintes solúveis e estruturais (Maurício et al., 2003).

A descrição matemática das curvas de produção de gases permite a análise dos dados, a comparação dos substratos e a evolução de diferentes ambientes de fermentação. Os dados obtidos proporcionam valiosa informação sobre a composição do substrato estudado e a taxa de fermentação dos constituintes solúveis e estruturais dos substratos. Os modelos matemáticos mais utilizados para estimar esta cinética são os de Ørskov e McDonald (1979), Mertens e Loften (1980), Beuvink e Kogut (1993), France et al. (1993), logístico uni ou bicompartimental, proposto por Schofield et al. (1994), Groot et al. (1996) e de Gompertz, proposto por Lavrencic et al. (1997).

O modelo de Gompertz supõe que a taxa de produção de gases é equivalente à atividade dos microrganismos, porém, uma vez aumentado o tempo de incubação, esta relação diminui, podendo ser subentendido que, ao longo do processo fermentativo, ocorre diminuição do potencial dos microrganismos (Noguera et al., 2004). O modelo logístico bicompartimental permite caracterizar os carboidratos de rápida e lenta degradação, fornecendo maiores informações a respeito do processo fermentativo.

Modelos que representam melhor conjunto de dados apresentam menor soma do quadrado do erro (SQE) (Noguera et al., 2004), e quanto menor o quadrado médio do erro (QME) melhor o ajuste do modelo, segundo Beuvink e Kogut (1993). Valor elevado do $\mathrm{R}^{2}$ do modelo pode indicar boa estimativa dos dados em relação aos valores observados, mas não deverá ser usado como único critério de seleção do modelo (Noguera et al., 2004).
Este trabalho foi realizado com o objetivo de comparar o modelo de Gompertz e o logístico bicompartimental na estimativa da cinética de fermentação ruminal dos carboidratos de coprodutos do babaçu (Orbignya martiana), pela técnica in vitro semiautomática de produção de gases.

\section{MATERIAL E MÉTODOS}

Foram realizados dois ensaios para se avaliar a capacidade de ajuste dos modelos de Gompertz e do logístico bicompartimental à estimativa da cinética de fermentação ruminal da matéria seca e da FDN do farelo e da torta de babaçu. As amostras do farelo e da torta do babaçu foram obtidas de vários fornecedores do estado do Piauí. Apenas a torta foi submetida à trituração em moinho tipo Willey com peneira de porosidade 1,0mm de diâmetro. Posteriormente, as amostras foram armazenadas em recipientes hermeticamente fechados.

Para avaliação dos modelos matemáticos, utilizou-se a técnica in vitro semiautomática de produção de gases, de acordo com Maurício et al. (2001). Foram incubadas amostras de farelo e torta de babaçu em inóculo ruminal. O inóculo foi coletado de um bovino adulto canulado no rúmen, às sete horas, antes do fornecimento da primeira refeição. As alíquotas das frações sólidas e líquidas do conteúdo ruminal foram coletadas diretamente do rúmen e acondicionadas em garrafas térmicas previamente aquecidas a $39^{\circ} \mathrm{C}$. O inóculo ruminal foi obtido após homogeneização, em liquidificador, das frações sólida e líquida do conteúdo ruminal, em igual proporção (1:1), seguida de filtragem. Filtrado, o inóculo foi mantido a $39^{\circ} \mathrm{C}$, sob contínua gaseificação com $\mathrm{CO}_{2}$ para manutenção da anaerobiose até o momento da incubação. As amostras foram incubadas utilizando-se frascos de vidro com capacidade de $160 \mathrm{~mL}$. Foram adicionados $1 \mathrm{~g}$ de amostra e $90 \mathrm{~mL}$ do meio de cultura, preparado segundo Maurício et al. (2001), e 10mL do inóculo ruminal. $\mathrm{O}$ meio foi gaseificado com $\mathrm{CO}_{2}$, os frascos foram vedados com rolhas de borracha e anilhas de alumínio e acondicionados em estufa com circulação forçada de ar a $39^{\circ} \mathrm{C}$. Frascos contendo somente meio de cultura e inóculo foram utilizados como controle. 
As leituras de pressão foram realizadas nos tempos de três, seis, nove, 12, 15, 21, 27, 33, 39, 48, 60, 72 e 96 horas após a incubação, com o auxílio de um transdutor de pressão conectado a uma válvula de três saídas, segundo Maurício et al. (2001). Para a estimativa do volume, utilizouse a equação de regressão: $\mathrm{V}=0,113 \mathrm{P}^{2}+3,8955 \mathrm{P}$ $\left(\mathrm{R}^{2}=0,99\right)$, em que $\mathrm{V}=$ volume em $\mathrm{mL}$ e $\mathrm{P}=$ pressão em psi.

Os modelos matemáticos avaliados estão descritos na Tab. 1. Para validação dos modelos, utilizou-se o quadrado médio do erro (QME), o coeficiente de determinação $\left(\mathrm{R}^{2}\right)$, o desvio médio absoluto dos resíduos (DMA) e a análise gráfica dos resíduos (Sarmento et al., 2006).
O QME foi obtido por meio da análise de variância, com o auxílio do procedimento PROC NLIN do SAS (1999), dividindo-se a SQE pelo número de observações (n). Para o R ${ }^{2}$, o DMA e a análise gráfica dos resíduos, utilizou-se o procedimento PROC CORR do SAS (1999). O $\mathrm{R}^{2}$ foi o resultado da divisão da soma do quadrado do modelo (SQM) pela soma do quadrado total (SQT). O DMA foi calculado a partir da diferença do somatório do valor observado pelo estimado, dividido pelo tamanho da amostra; quanto menor o valor do DMA melhor o ajuste (Sarmento et al., 2006). A dispersão dos dados foi estudada pela diferença dos valores observados e os estimados (Sarmento et al., 2006).

Tabela 1. Descrição matemática dos modelos avaliados

\begin{tabular}{|c|c|c|}
\hline Modelo & $\begin{array}{c}\mathrm{N}^{\mathrm{o}} \text { de } \\
\text { parâmetros }\end{array}$ & Equação ${ }^{1}$ \\
\hline Logístico bicompartimental & 5 & $\begin{array}{c}\mathrm{V}_{\mathrm{T}}=\mathrm{Vf} 1 /(1+\exp (2-4 * \mathrm{c} 1 *(\mathrm{~T}-\mathrm{L})))+\mathrm{Vf} 2 / \\
\left(1+\exp \left(2-4 *^{*} \mathrm{c} 2 *(\mathrm{~T}-\mathrm{L})\right)\right)\end{array}$ \\
\hline de Gompertz & 3 & $V_{T}=V_{F} x \exp \left(-C^{*} \exp (-A \times t)\right)$ \\
\hline \multicolumn{3}{|c|}{$\begin{array}{l}{ }^{1} \mathrm{~V}_{\mathrm{T}}=\text { volume total de gases (mL/100mg } \mathrm{MS} \text { ) acumulado no tempo } \mathrm{T} \text {; Vf1= volume final de gases oriundo da } \\
\text { degradação da fração solúvel de rápida digestão quando } \mathrm{T} \rightarrow \infty \text {; c1= taxa específica de produção de gases pela } \\
\text { degradação da fração solúvel; c2= taxa específica de produção de gases pela degradação da fração insolúvel; } \mathrm{T}= \\
\text { tempo de incubação (h); } \mathrm{L}=\text { fase de latência; } \mathrm{Vf} 2=\text { volume final de gases provenientes da degradação da fração } \\
\text { insolúvel de lenta digestão quando- } \mathrm{T} \infty ; \mathrm{V} \text { F = assíntota ou potencial máximo de produção de gases; } \mathrm{C}=\text { taxa } \\
\text { constante de produção de gases do material potencialmente degradável e } \mathrm{A}=\text { fator constante de eficiência microbiana. }\end{array}$} \\
\hline
\end{tabular}

\section{RESULTADOS E DISCUSSÃO}

Os valores obtidos para QME, R² e DMA estão apresentados na Tab. 2. O modelo logístico bicompartimental apresentou valor do QME bastante inferior ao de Gompertz, diferente dos resultados obtidos por Beuvink e Kogut (1993) e Noguera et al. (2004), com maior valor do QME para o modelo logístico em comparação ao modelo de Gompertz. Todavia, outros trabalhos corroboram os resultados encontrados neste trabalho. Barbosa (2007) obteve maior valor do QME para o modelo de Gompertz, em relação ao logístico, ao avaliar a cinética fermentativa de vagens de faveira e pau-ferro. Azevedo (2007), ao estudar a cinética fermentativa do pseudofruto do cajueiro, obteve, também, valor do QME mais alto para o modelo de Gompertz.

Tabela 2. Médias do quadrado médio do erro (QME), do coeficiente de determinação ( $\mathrm{R}^{2}$ ) e do desvio médio absoluto (DMA) obtidas a partir dos ajustes dos dados de produção de gases da matéria seca com os modelos logístico bicompartimental e de Gompertz

\begin{tabular}{lccc}
\multicolumn{1}{c}{ Modelo } & QME & $\mathrm{R}^{2}$ & DMA \\
\hline Logístico bicompartimental & 6,18 & 0,99 & 2,13 \\
de Gompertz & 21,26 & 0,99 & 3,79 \\
\hline
\end{tabular}

Os valores de $\mathrm{R}^{2}$ obtidos foram elevados $(0,99)$ e sem diferença $(\mathrm{P}>0,05)$ entre os modelos estudados. Barbosa (2007) também não observou diferença significativa quando comparou os modelos. No entanto, Azevedo (2007), apesar de ter encontrado $\mathrm{R}^{2}$ alto para todos os modelos estudados, observou que, para o modelo logístico, o $\mathrm{R}^{2}$ foi maior. Segundo Noguera et al. (2004), utilizar esse parâmetro como único critério de avaliação não é recomendado, pois 
altos coeficientes podem ser encontrados em modelos com limitada capacidade de predição.

O modelo logístico apresentou um valor de DMA menor em relação ao modelo de
Gompertz, resultado também obtido por Azevedo (2007) e Barbosa (2007).

Na Fig. 1, apresentam-se as dispersões temporais médias dos resíduos para os dois modelos.

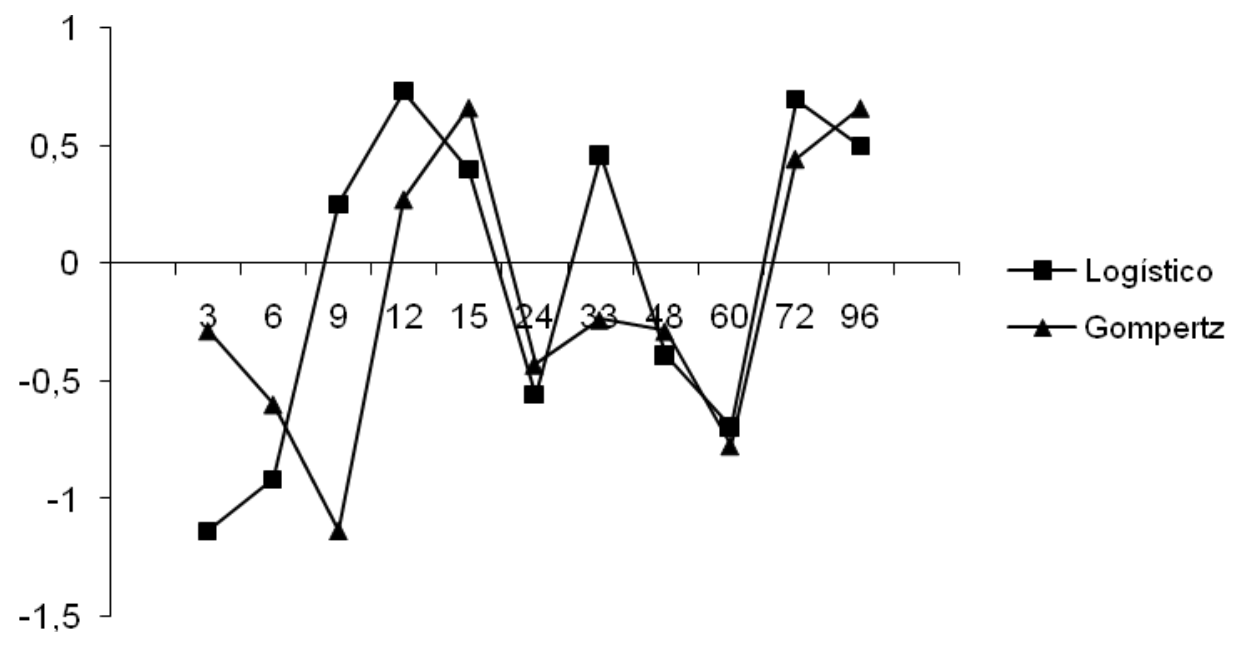

Figura 1. Dispersão temporal dos resíduos obtidos dos dados médios de produção de gases (mL gases/100 gMS) pelos modelos logístico e de Gompertz.

Para o modelo logístico, foi observado que, entre três horas e antes de completar nove, às 24 horas e entre 48 horas e antes de completar 72 horas, os valores foram superestimados. Entre nove e 15 horas, houve subestimativa. Às 33 horas e entre 72 e 96 horas, houve, também, subestimativa dos dados. Em nenhum momento a taxa de produção de gases predita foi igual à observada. Azevedo (2007) obteve menor dispersão temporal dos resíduos nos modelos logísticos e de Gompertz em relação a outros modelos estudados.

Quando utilizado o modelo de Gompertz, também foi observada variação no ajuste. Entre três horas até antes de 12 horas, 24 horas até antes de 72 horas aproximadamente de incubação, os valores foram superestimados. Subestimativas dos valores foram obtidas entre aproximadamente 12 horas às 24 horas e após 72 horas. Em nenhum momento os dados estimados foram iguais aos observados.

Com base nos gráficos de dispersões, pode-se verificar que tanto o modelo logístico como o modelo de Gompertz apresentaram bom resultado. Barbosa (2007) verificou que o modelo logístico foi o que melhor predisse as taxas de produção de gases, assim como Azevedo (2007). Resultado divergente foi obtido por Noguera et al. (2004), em que o melhor ajuste foi para o modelo de Gompertz em relação ao logístico, ao estudarem cinco genótipos de sorgo. Gonçalves et al. (2001), ao estudarem alguns volumosos na alimentação de cabras leiteiras, obtiveram resultados satisfatórios utilizando o modelo logístico.

Nas Fig. 2 e 3, apresentam-se a produção cumulativa de gases obtidos a partir dos dados observados e dos ajustados pelos dois modelos. Para estimativa da produção de gases, tanto o modelo logístico quanto o modelo de Gompertz podem ser utilizados, porém, de acordo com os parâmetros encontrados, o modelo logístico apresentou melhor valor de QME, critério que recomenda o seu uso. 


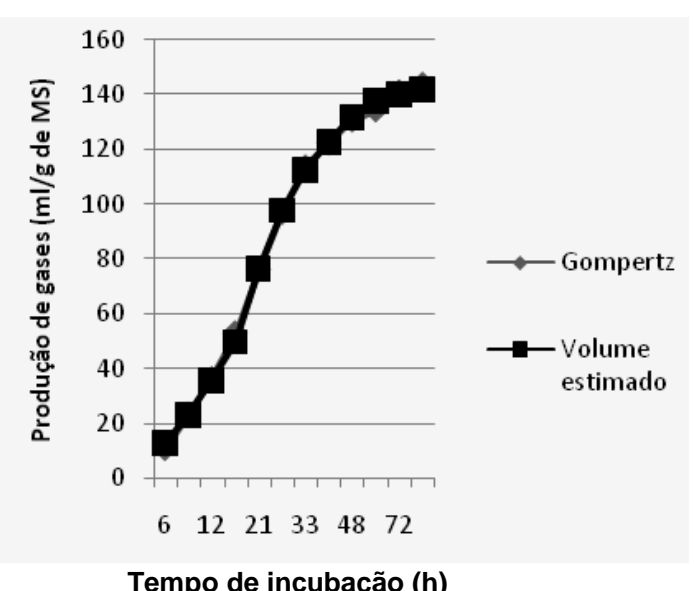

Figura 2. Curvas de produção cumulativa de gases da matéria seca do farelo e da torta de babaçu (Orbignya martiana) a partir da média dos dados observados e dos ajustados pelo modelo de Gompertz.

Barbosa (2007) obteve melhor ajuste para o modelo logístico em relação ao modelo de Gompertz, ao estudar a faveira e o pau-ferro. Azevedo (2007) encontrou, nos modelos logístico, exponencial e de France, ajuste adequado a todas as etapas do processo fermentativo, descrevendo as características de fermentação do material avaliado, tanto na fase inicial como na final.

$\mathrm{Na}$ análise gráfica dos resíduos, o modelo logístico subestimou apenas no final da curva e superestimou no início. As curvas de produção de gases caracterizam-se por apresentar forma sigmoidal, podendo ser distinguidas três fases: inicial de baixa produção, fase exponencial de rápida produção e uma fase assintótica de lenta ou inexistente produção de gases (Noguera et al., 2004). Nas primeiras horas de fermentação, uma parte do substrato, geralmente os açúcares solúveis, é fermentada rapidamente, mas isto representa uma pequena porção do total a ser degradado. Na sequência, uma menor quantidade de alimento é hidratada e colonizada pela microbiota, dando origem a degradações distintas, dependendo do substrato, quanto aos constituintes solúveis e estruturais (Beuvink e Kogut, 1993). Giraldo et al. (2006), em estudo sobre a relação entre pressão e volume para a

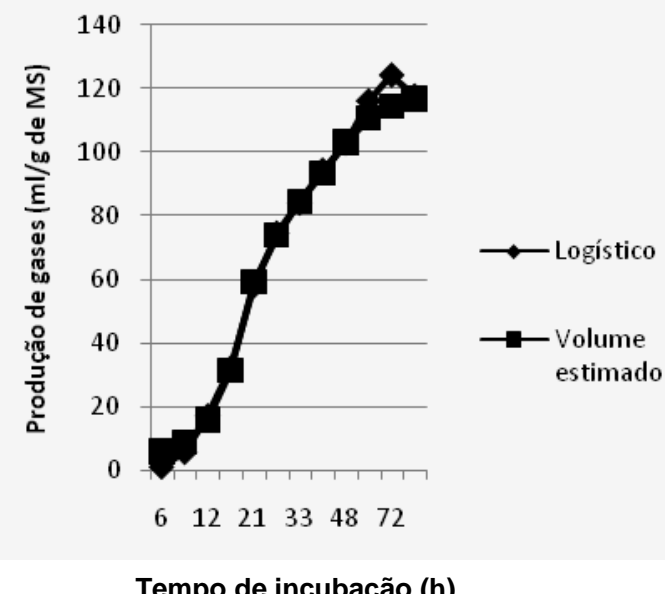

Figura 3. Curvas de produção cumulativa de gases da matéria seca do farelo e da torta de babaçu (Orbignya martiana) a partir da média dos dados observados e dos ajustados pelo modelo logístico bicompartimental.

implantação da técnica in vitro de produção de gases, na Colômbia, obtiveram taxas de fermentação mais altas nas primeiras horas de incubação e atribuíram o fato à fermentação dos carboidratos solúveis, e também observaram grande diferença entre os alimentos estudados. Verificaram, ainda, que, entre seis e 12 horas de incubação, houve aumento na taxa de produção de gases, que foi atribuído à fermentação de carboidratos fibrosos ou estruturais.

O QME, o $\mathrm{R}^{2}$ e o DMA dos modelos estudados para descrever a produção de gases oriundos da fermentação ruminal da FDN encontram-se na Tab. 3. Quanto ao QME, o modelo logístico bicompartimental foi o que apresentou menor valor. Os valores de $\mathrm{R}^{2}$ foram elevados $(0,99)$ para os dois modelos. Os desvios médios absolutos diferiram entre os modelos, com o de Gompertz apresentando valor mais elevado. Azevedo (2007) obteve valores de $\mathrm{R}^{2}$ baixos para todos os modelos, sendo o menor valor para o de Gompertz. Schofield et al. (1994), ao compararem alguns modelos para descrever a cinética da digestão da fibra, obtiveram valores elevados de $\mathrm{R}^{2}$ para o logístico bicompartimental e o de Gompertz, com menor valor para o primeiro. 
Tabela 3. Médias do quadrado médio do erro (QME), do coeficiente de determinação ( $\mathrm{R}^{2}$ ) e do desvio médio absoluto (DMA) obtidas a partir dos ajustes dos dados de produção de gases da parede celular com os modelos logístico e de Gompertz

\begin{tabular}{llll} 
Modelo & QME & $\mathrm{R}^{2}$ & DMA \\
\hline Logístico & 6,39 & 0,99 & 1,98 \\
de Gompertz & 11,1 & 0,99 & 3,21 \\
\hline
\end{tabular}

Nas Fig. 4 e 5, apresentam-se as produções cumulativas de gases obtidos a partir dos dados observados e dos ajustados pelos modelos de

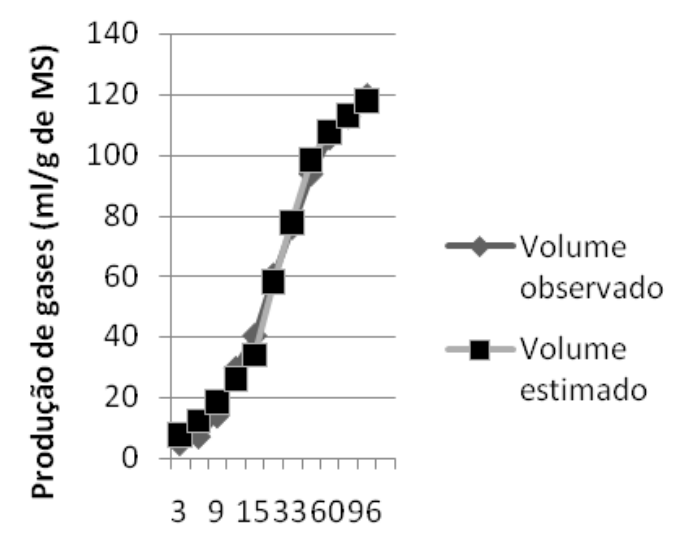

Tempo de incubação (h)

Figura 4. Modelo de Gompertz para o farelo e a torta de babaçu.

Azevedo (2007) obteve melhor ajuste para o modelo logístico bicompartimental e exponencial do que para os de France e de Gompertz, no entanto utilizou o modelo de Gompertz, que, apesar de ter apresentado maior dispersão do resíduo, assim como valores mais elevados para QME e DMA, apresentou melhor estimativa para a curva de produção de gases.

\section{CONCLUSÕES}

O modelo logístico bicompartimental estimou os valores de produção de gases melhor do que o modelo de Gompertz. Desse modo, pode ser adotado na estimativa da cinética de fermentação ruminal da matéria seca e fibra em detergente neutro do farelo e da torta de babaçu.

\section{AGRADECIMENTOS}

Ao Conselho Nacional de Desenvolvimento Científico e Tecnológico (CNPq), pela bolsa de estudo concedida e pelo apoio financeiro necessário à condução da pesquisa.
Gompertz e logístico bicompartimental para a FDN do farelo e da torta de babaçu.

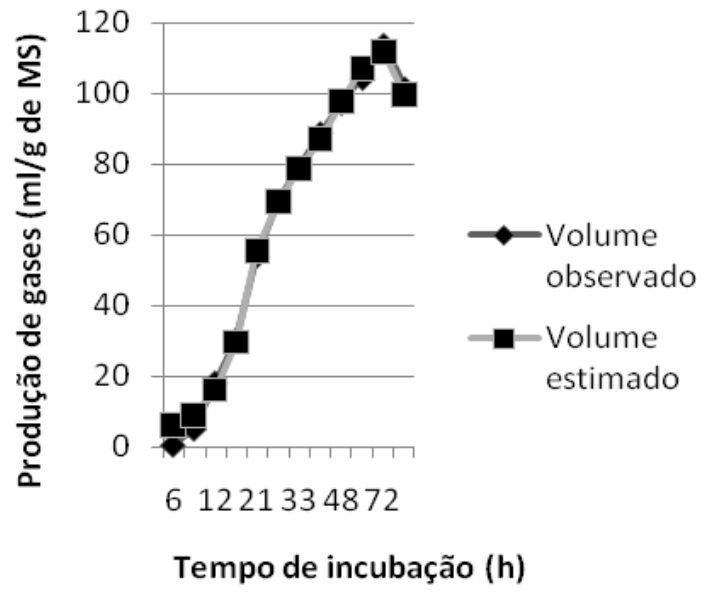

Figura 5. Modelo logístico para o farelo e a torta de babaçu.

\section{REFERÊNCIAS BIBLIOGRÁFICAS}

AZEVEDO, M.M.R. Parâmetros cinéticos da fermentação ruminal do pseudofruto de cinco clones de cajueiro pela técnica in vitro semiautomática de produção de gases. 2007. 44f. Dissertação (Mestrado) - Universidade Federal do Piauí, Teresina.

BARBOSA, A.L. Valor nutritivo de vagens de faveira (Parkia platycephela Benth.) e pau-ferro (Caesalpinea ferrea Mart. Ex Tul.) através da técnica in vitro semiautomática de produção de gases. 2007. 44f. Dissertação (Mestrado) Universidade Federal do Piaú, Teresina.

BEUVINK, J.M.W.; KOGUT, J. Modeling gas production kinetics of grass silages incubated with buffered ruminal fluid. J. Anim. Sci., v.71, p.1041-1046, 1993.

FRANCE, J.; DHANOA, M.S.; THEODOROU, M.K. et al. A model to interpret gas accumulation profiles associated with in vitro degradation of ruminant feeds. J. Theoret. Biol., v.163, p.99-111, 1993. 
GIRALDO, L.A.; GUTIÉRREZ, L.A.; SÁNCHEZ, J. et al. Relación entre presión y volumen para el montaje de la técnica in vitro de producción de gas em Colombia. Livestock Research for Rural Development, 2006. Disponível em: <http://www.cipav.org.co/1rrd/1rrd18/6/gira1807 5.htm/>. Acessado em: 26 ago 2007.

GONÇALVES, A.L.; LANA, R.P.; RODRIGUES, M.T. et al. Cinética de degradação de alguns volumosos usados na alimentação de cabras leiteiras por intermédio da técnica de produção de gases sob diferentes níveis de pH. Rev. Bras. Zootec., v.30, p.19041912, 2001.

GROOT, J.C.J.; CONE, J.W.; WILLIAMS, B.A. et al. Multiphasic analysis of gas production kinetics for in vitro fermentation of ruminant feeds. Animal Feed Science and Technology, v.64, p.77-89, 1996.

JAYME, D.G.; GONÇALVES, L.C.; MAURÍCIO, R.M. et al. Avaliação pela técnica de produção de gases das silagens de quarto genótipos de girassol (Helianthus annuus) (Rumbosol 91, Victoria 627, Victoria 807 e Mycogen 93338). Arq. Bras. Med. Vet. Zootec., v.61, p.1403-1410, 2009.

LAVRENCIC, A.; STEFANON, B.; SUSMEL, $P$. An evaluation of the Gompertz model in degradability studies of forage chemical components. Anim. Sci., v.64, p.423-431, 1997.

MAURICIO, R.M.; OWEN, E.; MOULD, F.L. et al. Comparison of bovine rumen liquor and bovine faeces as inoculum for an in vitro gas production technique for evaluating forages. Anim. Feed Sci. Technol., v.89, p.33-48, 2001.
MAURÍCIO, R.M.; PEREIRA, L.G.R.; GONÇALVES, L.C. et al. Potencial da técnica in vitro semiautomática de produção de gases para avaliação de silagens de sorgo (Sorghum bicolor (L.) Moench). Rev. Bras. Zootec., v.32, p.10131020, 2003.

MERTENS, D.R.; LOFTEN, J.R. The effect of starch on forage fiber digestion kinetics in vitro. J. Dairy Sci., v.63, p.1437-1446, 1980.

NOGUERA, R.R.; SALIBA, E.O.; MAURICIO, R.M. Comparación de modelos matemáticos para estimar los parámetros de degradación obtenidos a través de la técnica de producción de gas. 2004. Disponível em: <http://www.cipav.org.co/1rrd/1rrd16/11/nogu16 086.htm/>. Acessado em: 3 abr 2006.

ØRSKOV, E.R.; McDONALD, I. The estimation of protein degradability in the rumen from incubation measurements weighted according to rate of passage. J. Agric. Sci., v.92, p.499-503, 1979.

SARMENTO, J.L.R.; REGAZZI, A.J.; SOUSA, W.H. et al. Estudo da curva de crescimento de ovinos Santa Inês. Rev. Bras. Zootec., v.35, p.435-442, 2006.

SCHOFIELD, P.; PITT, R.E.; PELL, A.N. Kinetics of fiber digestion from in vitro gas production. J. Anim. Sci., v.72, p.2980-2991, 1994. 